\title{
Tackling penicillin allergy delabeling with ultra-sensitive in vitro test and human-like specific IgE standards
}

\author{
Sergi Morais ${ }^{1}$, Pedro Quintero-Campos ${ }^{1}$, Paula Segovia ${ }^{2}$, Ethel Ibáñez-Echevarria ${ }^{3}$, Dolores \\ Hernández-Fernández de Rojas ${ }^{4}$, Patricia Casino ${ }^{5}$, Gabriel Lassabe ${ }^{2}$, Gualberto \\ González-Sapienza $^{2}$, and Ángel Maquieira ${ }^{1}$ \\ ${ }^{1}$ Instituto Interuniversitario de Investigación de Reconocimiento Molecular y Desarrollo \\ Tecnológico (IDM) Universitat Politècnica de València-Universitat de València Spain \\ ${ }^{2}$ Cátedra de Inmunología Facultad de Química DEPBIO Instituto de Higiene Montevideo \\ Uruguay \\ ${ }^{3}$ Hospital Universitari I Politènic La Fe Servicio de Alergia \\ ${ }^{4}$ Allergy Therapeutics Ibérica Av de Barcelona 11508970 Sant Joan Despí Spain \\ ${ }^{5}$ Group 739 of the Centro de Investigación Biomédica en Red sobre Enfermedades Raras \\ (CIBERER) del Instituto de Salud Carlos III Spain
}

December 3, 2021

\begin{abstract}
Background: Penicillin allergy delabeling initiatives are now part of antibiotic stewardship programs and include the use of invasive and risky in vivo tests. Instead, the quantification of specific IgE is highly useful to confirm immediate allergy to penicillins. However, discrepant results associated to the low sensitivity of the in vitro tests have limited their routine diagnostic use for delabeling purposes. We aimed to tackle a novel diagnostic strategy for specific IgE testing based on a homologous interpolation scheme, using recombinantly produced standards. Methods: Serum samples from a cohort of allergic patients and controls were analysed by a chemiluminescence-based immunoassay, using a bispecific binanobody as standard. The novel standard targets the major antigenic determinant of penicillin G and the paratope of Omalizumab, acting as human-like specific IgE. Results: Testing a cohort of 65 human serum samples, the method achieved a good agreement and strong positive relationship, reaching a limit of detection below $0.1 \mathrm{IU} / \mathrm{mL}$. The sensitivity of the in vitro test significantly increased (66 \%), doubling that of the ImmunoCAP reference in vitro assay with an overall specificity of $100 \%$. Conclusions: The new diagnostic strategy compares favourably with the results obtained on the ImmunoCAP system, paving the way towards the standardization of penicillin allergy testing. The recombinant standards are potent calibrators, highly stable, easy and inexpensive to produce, and overcome the limitation associated with preparations derived from pooled human serum, expediting the production of next generation standards with different specificities to successfully tackle $\beta$-lactam allergy delabeling by in vitro tests.
\end{abstract}

Tackling penicillin allergy delabeling with ultra-sensitive in vitro test and human-like specific IgE standards

Short title: Recombinant standard for in vitro penicillin allergy testing

Pedro Quintero-Campos ${ }^{1}$, Paula Segovia ${ }^{2}$, Ethel Ibáñez-Echevarria ${ }^{3}$, Sergi Morais ${ }^{* 1,4,5}$ Dolores HernándezFernández de $\operatorname{Rojas}^{6}$, Patricia Casino ${ }^{7,8,9}$ Gabriel Lassabe ${ }^{2}$, Gualberto González-Sapienza*2, Ángel Maquieira*1,4,5

${ }^{1}$ Instituto Interuniversitario de Investigación de Reconocimiento Molecular y Desarrollo Tecnológico (IDM), Universitat Politècnica de València-Universitat de València, Spain. 
${ }^{2}$ Cátedra de Inmunología, Facultad de Química, DEPBIO, Instituto de Higiene, Montevideo, Uruguay.

${ }^{3}$ Hospital Universitari I Politènic La Fe, Servicio de Alergia, ${ }^{4}$ Unidad Mixta UPV-La Fe, Nanomedicine and Sensors, IIS La Fe, Av. de Fernando Abril Martorell, 106, 46026 València, Spain.

${ }^{5}$ Departamento de Química, Universitat Politècnica de València, Camino de Vera s/n, 46022 Valencia, Spain.

${ }^{6}$ Allergy Therapeutics Ibérica. Av. de Barcelona, 115, 08970, Sant Joan Despí, Spain.

${ }^{7}$ Departamento de Bioquímica y Biología Molecular, ${ }^{8}$ Instituto Universitario de Biotecnología i Biomedicina (BIOTECMED), Universitat de València. Dr Moliner 50, 46100, Burjassot, Spain.

${ }^{9}$ Group 739 of the Centro de Investigación Biomédica en Red sobre Enfermedades Raras (CIBERER) del Instituto de Salud Carlos III, Spain.

*Corresponding author: Sergi Morais (ORCID ID: 0000-0002-3722-2358)

\section{Acknowledgments}

P.Q-C acknowledges financial support from Generalitat Valenciana through the research staff-training program (GVA ACIF / 2018/173). P.C. awards grants Ramón y Cajal and PID2019-110630GB-I00 funded by MCIN/AEI/ 10.13039/501100011033 and by "ERDF A way of making Europe". S.M awards grants PID2019110713RB-I00 funded by MCIN/AEI/ 10.13039/501100011033 and by "ERDF A way of making Europe". PROMETEO/2020/094, Proyecto "88/2021" "Nominativa Acción 2021-12. $\beta$-DesLac funded by Generalitat Valenciana, FMV-ANII 148245, FMV-ANIII 156321 funded by Agencia Nacional de Investigación and CSIC 2007-348 UdelaR, Uruguay, are also acknowledged..

P. Q-C performed experiments, analyzed the data, wrote and read the manuscript; P.S analyzed the data and read the manuscript; E. I-E and D. H-F collected the patients' samples and contributed to the clinical investigation of the patients and read the manuscript; P. C designed and supervised the experiments and read the manuscript; S. M designed and supervised experiments, analyzed the data, wrote and read the manuscript; G. L and G. G-S supervised the experiments and read the manuscript; Á. M supervised experiments and read the manuscript.

\section{Abstract}

Background: Penicillin allergy delabeling initiatives are now part of antibiotic stewardship programs and include the use of invasive and risky in vivo tests. Instead, the quantification of specific IgE is highly useful to confirm immediate allergy to penicillins. However, discrepant results associated to the low sensitivity of the in vitro tests have limited their routine diagnostic use for delabeling purposes. We aimed to tackle a novel diagnostic strategy for specific IgE testing based on a homologous interpolation scheme, using recombinantly produced standards.

Methods : Serum samples from a cohort of allergic patients and controls were analysed by a chemiluminescence-based immunoassay, using a bispecific binanobody as standard. The novel standard targets the major antigenic determinant of penicillin $\mathrm{G}$ and the paratope of Omalizumab, acting as human-like specific IgE.

Results : Testing a cohort of 65 human serum samples, the method achieved a good agreement and strong positive relationship, reaching a limit of detection below $0.1 \mathrm{IU} / \mathrm{mL}$. The sensitivity of the in vitro test significantly increased $(66 \%)$, doubling that of the ImmunoCAP reference in vitro assay with an overall specificity of $100 \%$.

Conclusions: The new diagnostic strategy compares favourably with the results obtained on the ImmunoCAP system, paving the way towards the standardization of penicillin allergy testing. The recombinant standards are potent calibrators, highly stable, easy and inexpensive to produce, and overcome the limitation 
associated with preparations derived from pooled human serum, expediting the production of next generation standards with different specificities to successfully tackle $\beta$-lactam allergy delabeling by in vitrotests.

Keywords: allergy, $\beta$-lactam antibiotics, binanobody, diagnostics, Omalizumab.

\section{Introduction}

Currently over 31 million of the EU-27 population (6.9\%) carry a $\beta$-lactam allergy label, which prevents them from receiving the first-line antibiotics such as penicillin. This contributes to the prescription of antibiotics that diverge from the guidelines, increasing antimicrobial resistance, one of the biggest threats to global health. Delabeling initiatives are now part of antibiotic stewardship programs and include the use of invasive, time-consuming and risky in vivo tests. The quantification of specific IgE to penicillins is highly valuable for allergy monitoring, confirming or ruling out immediate allergy and finding safe alternatives, however, the current in vitro tests lack sensitivity (ca. $75 \%$ false negatives), which limits their routine diagnostic use for delabeling purposes.

In vitro diagnosis of human allergies is based on the immunoanalytical quantification of specific $\operatorname{IgE}(\mathrm{sIgE})$ in serum ${ }^{1}$. Currently, several assays are commercially available for the determination of sIgE, as ImmunoCAP (Thermo Fisher Scientific), Immulite and Atellica (Siemens), Euroline (Euroimmun), Noveos (Hycor), ALEX2 (Macro Array Diagnostics) ${ }^{2}$ and ALFA (Dr. Fooke) ${ }^{3}$. However, scientific evidence has shown that the results provided by these methods are not comparable with each other ${ }^{4}$, so there is an urgent need to harmonize protocols for the in vitro diagnosis of allergy.

The lack of standardized allergen-specific IgE reference preparations is one of the weaknesses of in vitro diagnosis $^{5}$. The concentrations of $\operatorname{sIgE}$ are calculated from a total $\mathrm{IgE}$ calibration curve by a heterologous interpolation. To do this, a set of calibrators (WHO 11/234) is used to transform the assay signals into quantitative allergen-specific $\mathrm{IgE}$ antibody units $(\mathrm{IU} / \mathrm{mL})^{6}$. However, recent studies have revealed a better correlation among methods when homologous calibration is used because of the significant differences found between the interpolation schemes for quantifying allergen-specific IgE. ${ }^{7,8}$ Therefore, the absence of internationally standardized allergen-specific IgE reference preparations is one of the reasons for the discrepant results between methods and probably the most important unresolved issue to harmonizein vitro serological quantitative allergy diagnostics.

The standard for the homologous calibration is the sIgE present in serum. Serum samples, however, show variability in terms of sIgE level, the concentration to $\beta$-lactam antibiotics being very low ${ }^{9}$. Furthermore, the sample volume is limited, its accessibility difficult and expensive as well. So, standardized reference material is currently not available. Besides, the use of positive human sera is key to perform the so-called "round robin" tests to evaluate the accuracy and precision (repeatability and reproducibility) of the in vitro allergy tests between laboratories.

The first attempt approaching synthetic standards consisted on raising specific antibody molecules by hyperimmunization to produce a fully humanized chimera ${ }^{10}$. Later, these molecules were produced recombinantly by binding the Fab to the human Fc region ${ }^{11,12}$. An advantageous alternative to conventional antibodybased standards is the use of nanobodies. These are recombinant antibody fragments $(15 \mathrm{kDa})$ derived from the variable domain $(\mathrm{VHH})$ of a particular type of antibodies found in camelids that are devoid of light chain ${ }^{13}$. They have remarkable physicochemical properties including thermal stability, solubility and are produced with high yields by bacterial fermentation. In addition, their monodomain nature facilitates the construction of phage display libraries, which offers the possibility of isolating nanobodies with the desired properties ${ }^{14}$. Thus, synthetic standards consisting of a nanobody against the target antigen expressed in tandem with a second nanobody against the paratope of the detection antibody can function as highly standardized surrogate homologous calibrators for the currently existing in vitro tests.

This study reports on the clinical evaluation of the ultra-sensitive chemiluminescence immunoassay, using synthetic bispecific binanobodies ( $\mathrm{rBBN}$ ) as the human IgE-like standard for the reliable quantification of IgE to penicillin $\mathrm{G}$ in a cohort of 65 human serum samples. The results were compared with those obtained 
with the ImmunoCAP system.

\section{Materials and methods}

Reagents, buffers, and consumables

Keyhole limpet hemocyanin (KLH), human serum albumin (HSA), histone H1, penicillin G (PG), bovine serum albumin (BSA), D-biotin, isopropyl $\beta$-D-1-thio-galactopyranoside (IPTG), polyethylene glycol 8000 (PEG), LB Broth (Miller), 3,3',5,5'- tetramethylbenzidine (TMB), tris base, Tween 20 and other chemicals were from Sigma (St. Louis, MO, USA).

Anti-hemagglutinin epitope (anti-HA) antibody conjugated to horseradish peroxidase (HRP) was from Sigma (Cat no. 12013819001). Omalizumab was from Novartis International AG (Basel, Switzerland). Anti-human IgG antibody-HRP (Cat No. ab97225) and the HRP Conjugation Kit (ab102890), the latter used to prepare Omalizumab-HRP, were from Abcam (Cambridge, UK).

E. coli One Shot BL21 (DE3) cells were from Thermo Fisher Scientific (Fremont, CA, USA). E. coli ER2738 electrocompetent cells were purchased from Lucigen Corporation (Middleton, WI, USA) and chromatography columns were from GE Healthcare (Piscataway, NJ, USA). High binding polystyrene ELISA plates were from Costar Corporation (Cambridge, MA, USA) and SuperSignal ELISA Femto Substrate, PCR Plate 96-well, and SYPR Orange Protein Gel Stain were acquired from Thermo Fisher (Madrid, Spain). Coating buffer

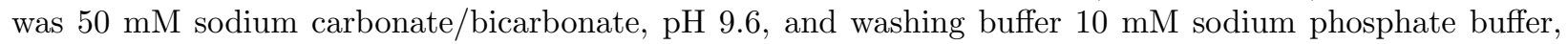
$150 \mathrm{mM} \mathrm{NaCl}, 0.05 \%$ Tween 20\%, pH 7.4 (PBS-T).

\section{Phage Display}

An adult llama (Lama glama) from the municipal zoo of Montevideo was immunized subcutaneously with 4 doses of $500 \mu \mathrm{g}$ of HSA-PG conjugate in Freund's Incomplete Adjuvant. Ten days after the last immunization, $200 \mathrm{~mL}$ of blood was drawn. From the extracted blood, lymphocytes were purified by gradient centrifugation with Histopaque (Sigma) according to the manufacturer's protocol. From $10^{8}$ cells, total RNA was extracted using TRIZOL (Invitrogen, Carlsbad, CA USA), and retrotranscribed to cDNA using the RevertAID Reverse Transcriptase (Thermo Fisher Scientific, Carlsbad, CA, USA). Subsequently, the DNA fragments encoding the $\mathrm{VH} / \mathrm{VHH}$ of the IgGs were amplified by PCR as described in the supporting information. The fragments obtained were digested using SfiI, cloned into the pComb3X phagemid vector (from Dr. Barbas, The Scripps Research Institute, La Jolla, USA) and electroporated into E. coli ER2738 competent cells. Finally, cells were cultured and superinfected with the helper phage M13KO7 to generate the phage library. Next, two rounds of panning were performed. The eluted phages were used to infect ER2738 cells and grown on $\mathrm{LB}$ /agar plates. From the isolated bacterial colonies, $2 \mathrm{~mL}$ cultures of each clone were grown in $\mathrm{SB}$ with 0.1 $\mathrm{mg} / \mathrm{mL}$ ampicillin at $37{ }^{\circ} \mathrm{C}$ and shaking until an optical density of 0.6 was reached. After that, nanobody expression was induced by the addition of $1 \mathrm{mM}$ IPTG and incubated with shaking at $37{ }^{\circ} \mathrm{C}$ for 16 hours. After centrifugation, the supernatants, containing the nanobodies, were collected and tested in ELISA plates coated with KLH-PG conjugate. Bound nanobodies were detected with an anti-HA-HRP conjugate following the protocol described in the supporting information.

Cloning, expression, and purification of $r B B N$.

Clones that were specific to the PG antigen were selected and the VHH genes amplified and subcloned into the pINQ-bis vector. This vector is an in house modified version of the pET-28a $(+)$ vector that allows the expression of bispecific nanobodies in the periplasm of E. coliBL21(DE3). The modification resulted in an expression cassette coding for ompA signal peptide, the selected anti- $\beta$-lactam VHH flanked by two SfiI restriction sites, and a spacer of GQAGR(GGGGS)3TSEL, followed by an anti-omalizumab VHH sequence and a $6 \mathrm{xHis}$ tag. The vector was transformed into E. coli strain (DE3). Individual clones isolated on LBkanamycin plates were grown in $500 \mathrm{~mL}$ of LB with kanamycin $(50 \mu \mathrm{g} / \mathrm{mL})$ and induced with $10 \mu \mathrm{M}$ IPTG for 16 hours at $28{ }^{\circ} \mathrm{C}$ under shaking.

Cultures were centrifuged and periplasmic proteins extracted by osmotic shock according to previously 
established protocols ${ }^{15}$. Then, rBBN were purified on $1 \mathrm{~mL}$ His-Trap columns on the ÄKTA purification system (GE Healthcare, Uppsala, Sweden) according to the manufacturer's instructions. The imidazoleeluted fractions were dialyzed against PBS and quantified spectrophotometrically (Abs $280 \mathrm{~nm} 0.1 \%=1.9$ ) and by Bradford's protein assay ${ }^{16}$.

\section{Biochemical characterization of $r B B N$}

The purified rBBN was analyzed by $12 \%$ sodium dodecylsulfate-polyacrylamide gel electrophoresis (SDSPAGE) and Coomassie brilliant blue staining method. The specificity and functionality of the rBBN was evaluated by ELISA, blue native PAGE and ThermoFluor assay as described in the supporting information.

Analysis of serum samples.

Serum samples from 65 adults were collected in red-top tubes (BD Diagnostics, Madrid, Spain) and incubated at room temperature for 60 minutes to induce clotting. After centrifugation at $2000 \mathrm{rpm}$ for 15 minutes, the serum was aliquoted into cryovials and stored at $-80^{\circ} \mathrm{C}$ until use. A cohort of 35 allergic patients who report a history of penicillin allergy confirmed by in vivo tests and 30 non-allergic individuals were included in the case-control study. The clinical history included also the concentration of sIgE measured by the ImmunoCAP system. This study was approved by the Hospital Universitari i Politècnic La Fe (Valencia, Spain) ethical review committee (registry no. COBIOPHAD). Signed informed consent were collected from the participants.

The samples were analysed by the homologous chemiluminescence immunoassay (hmCLIA). Briefly, a white flat-bottomed polystyrene ELISA plate was coated with H1-PG conjugate solution $(3 \mu \mathrm{g} / \mathrm{mL})$ in coating buffer. The next day, the plate was washed 4 times with PBS-T. After that $25 \mu \mathrm{L}$ of serum sample was added to each well, followed by incubation for $30 \mathrm{~min}$ at room temperature. The calibration curve was made using the produced synthetic standards diluted in a pooled control sIgE-free human serum. All serum samples were analysed in triplicate. Subsequently, after washing the plate, $25 \mu \mathrm{L} /$ well of Omalizumab-HRP solution (1/10,000 dilution) was incubated as before. After, peroxidase activity was measured by adding $25 \mu \mathrm{L}$ of enhanced chemiluminescent substrate solution previously diluted 1/10 in PBS. The luminescent signals were read at $450 \mathrm{~nm}$, using the EnSpire Multimode Plate Reader (PerkinElmer, Waltham, USA). The limit of detection (LOD) and limit of quantification (LOQ) were calculated by measuring ten times the blank signal and interpolating the mean signal plus 3 and 10 times the standard deviation to the calibration curve, respectively. Allergen-specific IgE levels expressed in PSU/mL (Penicillin Standardised Unit) were determined by interpolating the luminescent signals into the calibration curve.

\section{Statistical analysis}

Coefficients of variation and the parameters to evaluate the assay sensitivity were calculated by standard descriptive statistical methods using Microsoft Excel 365 (Microsoft Corporation). The standard data points were plotted and a four-parameter logistic (4PL) curve was fitted through the points, using SigmaPlot 12 (Systat Software Inc). ANOVA statistical analysis, multiple regression analysis and the correlation study were carried out using SPSS Statistics (IBM). Clinical sensitivity and specificity were calculated using MedCalc (MedCalc Software) using ImmunoCAP as reference method.

\section{Results and discussion}

Phage display.

The antibody titter of the llama serum, as it can be observed in Figure S1, was high after the fourth boost, revealing the production of specific antibodies to PG. Next, a phage-display VHH library with a size of $1 \times 10^{8}$ transformants was constructed from the lymphocytes of the immunized llama (see supporting information). Ten individual clones were selected and their reactivity was tested against a panel of antigen determinants to $\beta$-lactam antibiotics. As is shown in Figure S2, seven out of ten clones were specific to penicillin G (PG1-5, PG7-8), two clones were generic to the panel of determinants (PG9-10) and one was a nonspecific clone (PG6) as it showed reactivity against the carrier protein KLH. The PG8 clone, the best in performance, was selected for further cloning an expression. 
Cloning, expression, and purification of $r B B N$.

After subcloning into the pINQ-bis vector, sequencing confirmed that the clones possessed the two VHHs sequences of interest. Figure 1A depicts the structural model of rBBN, using the Robetta web server and visualized in UCSF Chimera. The model shows the two nanobodies connected by a loop (yellow); the $17 \mathrm{kDa}$ one (blue) targets the penicillin G determinant and the $18 \mathrm{kDa}$ one (green) the paratope of Omalizumab. Purified rBBN was analyzed by $12 \%$ SDS-PAGE gel electrophoresis, and a clear and intense band was observed (lane 3, Figure 1B), corresponding to the synthetic standard with an expected molecular weight around $35 \mathrm{kDa}$.

\section{Biochemical characterization of $r B B N$}

The functionality of rBBN was tested by ELISA, following the protocol described in the supporting information. As it can be seen in Figure 2A, the set of data fitted well to a four-parameter logistic model, revealing the standard was functional, as it targeted both the antigenic determinant to penicillin $\mathrm{G}$ and the paratope of Omalizumab. According to the lowest detectable concentration, the limit of detection was $0.13 \mathrm{ng} / \mathrm{mL}$ $(3.7 \mathrm{pM})$, under the ELISA conditions.

We also conducted a thermofluor assay to check if interaction of rBBN with H1-PG stabilized the complex. As it can be observed in Figure 2B, the Tm of rBBN alone is $59{ }^{\circ} \mathrm{C}$ while the Tm of rBBN in the presence of H1-PG increased around seven-Celsius degrees. This indicates that the rBBN-Penicillin G complex is more stable than rBBN alone, demonstrating the specific binding between the nanobody and the ligand.

The selectivity of rBBN for H1-PG and Omalizumab was also studied by Blue Native-PAGE, as rBBN has a high isoelectric point $(\mathrm{pI}=8.5)$ which prevented the protein from entering the gel in a regular Native-PAGE. In this way, tested proteins were mixed with a loading buffer containing Coomassie Blue G (Serva, GmbH) which adds negative charges to the proteins to force them entering the gel and running according to their molecular weight (MW) and hydrodynamic conformation. In Figure 2C, the electrophoretic mobility of the proteins alone and in complex is shown. The rBBN at 1 and $3 \mu \mathrm{M}$ run as a single band with high MW $\left({ }^{\sim} 245\right.$ $\mathrm{kDa}$ ), possibly due to the high hydrodynamic volume caused by the conformational flexibility of the loop connecting the two nanobodies. In the case of the H1-PG conjugate, three bands were shown, one at $~ 55$ $\mathrm{kDa}$, which we identified as the H1-PG conjugate monomer, a faint band around $\sim 100 \mathrm{kDa}$, which could be ascribed to a dimer and another at high MW ( $245 \mathrm{kDa}$ ) ascribed to multimers. Then, rBBN and H1-PG were mixed at a molar ratio of 1:1 (lane 4) and 1:3 (lane 5) during 15 minutes and were run in the gel. A decrease in the intensity for the $55 \mathrm{kDa}$ band of $\mathrm{H} 1-\mathrm{PG}$ was observed as the amount of rBBN increased, while strong accumulation around the higher MW band was shown, thus, ascribing the electrophoretic mobility changes to the complex formation. When Omalizumab was assayed, it barely entered the gel producing two faint bands at $\sim 150 \mathrm{kDa}$ and at $\sim 245 \mathrm{kDa}$, which could be ascribed to the monomeric and dimeric antibody respectively, and a strong accumulation of protein at the top of the gel that was ascribed to multimers. The addition of rBBN to Omalizumab was concomitant with the disappearance of the $150 \mathrm{kDa}$ band and a decreased intensity for the accumulated band which indicated complex formation. Finally, incubation of the three samples, H1-PG/rBBN/Omalizumab was performed at molar ratio of 1:1:1 and 1:3:1. As observed before, the band of $150 \mathrm{kDa}$ corresponding to H1-PG disappeared and a strong accumulation for the high MW band $(245 \mathrm{kDa})$ was shown. Interestingly, the Omalizumab band at the top of the gel dramatically decreased intensity and became diffused. These changes in the electrophoretic mobility are indicative of complex formation.

Analysis of serum samples.

The produced $\mathrm{rBBN}$ was used as a standard to determine the concentration of sIgE to penicillin G present in a representative serum sample, following the homologous calibration scheme (Figure 3A) in the hmCLIA method. The current method for the determination of sIgE follows a heterologous scheme and defines 1.0 $\mathrm{IU} / \mathrm{mL}$ as equivalent to $2.4 \mathrm{ng} / \mathrm{ml} \mathrm{IgE}$. Due to the remarkable difference in molecular weight between the international standard IgE (190 kDa) and rBBN (35 kDa), a new unit (Penicillin Standardised Unit, PSU), equivalent to $0.44 \mathrm{ng} / \mathrm{mL}$ of $\mathrm{rBBN}$ was established on the basis of the IgE-rBBN molar ratio that is 5.4. To 
build the calibration curve, rBBN was diluted in sIgE-free serum at concentrations of 95, 54, 19, 5.4, 3.8, 1.90 and $0.5 \mathrm{PSU} / \mathrm{mL}$. The stability of rBBN in sIgE-free human was studied for a period of $8 \mathrm{~h}$. The results revealed that there were no statistically significant differences $(\mathrm{p}<0.0001)$ between the signals obtained over the studied period of time (1-8 h), which guarantees its good performance during the total assay time.

Figure $3 \mathrm{~B}$ shows the calibration curve. The signals $(\mathrm{n}=10)$ were fitted to a four-parameter logistic curve, giving a dynamic response ranging from 0.1 to $95 \mathrm{PSU} / \mathrm{mL}$, and a LOD of $0.02 \mathrm{PSU} / \mathrm{mL}(8.8 \mathrm{pg} / \mathrm{mL})$, a concentration below the currently internationally accepted cut-off for allergy diagnosis $(0.1 \mathrm{IU} / \mathrm{mL}$ or 240 $\mathrm{pg} / \mathrm{mL}$ ). In the hmCLIA accuracy assessment, the CVs range from 2 to $9 \%$, resulted in a linear regression equation with a correlation coefficient ( $\mathrm{r}$ ) of 1 . Therefore, the results reveal that the hmCLIA assay, using the new standards under a homologous calibration, has good analytical performances.

A cohort of 65 sera collected from patients allergic to penicillin G (35) and non-allergic subjects (30) was analysed in triplicate by hmCLIA assay. The signals obtained for each of the samples were interpolated in both the homologous (synthetic standard) and heterologous (WHO international standard) calibration curves. Table 1 shows the results. The Pearson's correlation coefficient statistical study between the results obtained using both calibration methods (Figure 4A) displays that there is a direct positive correlation $(\mathrm{r}$ $=0.995, \mathrm{p}<0.0001)$ between the two interpolation schemes. Furthermore, the results obtained under the homologous calibration relate well with those obtained by ImmunoCAP, showing a Pearson correlation close to $1(\mathrm{r}=0.999, \mathrm{p}<0.0001)$ (Figure 4B). In addition, as it is shown in Figure 4B, the slope of the curve is 3 , representing the relationship between the value given by ImmunoCAP and that obtained using $\mathrm{rBBN}$ as standard.

To normalize the results, the concentrations given by the hmCLIA assay are multiplied by a factor of $1 / 3$ to get those obtained by ImmunoCAP (see results in Table 1). The use of this factor allows the classification of patients in the same RAST group. In comparison with the reference approach, the results using the new standard also showed recovery values ranging from 91 to 135\%, revealing a reliable quantification.

The linearity of dilution test was performed using a pooled positive serum sample (sample 36 in Table 1). The linearity dilution study was carried out using 4-fold serial dilutions (1/4-1/4096) prepared in the pooled control serum. The experimental concentrations were calculated by ImmunoCAP and hmCLIA. As is shown in Figure 4C, the linearity of the hmCLIA assay was good over a wide range of dilution, detecting sIgE up to the dilution $1 / 1024(0.04 \mathrm{PSU} / \mathrm{mL})$ what revealed good flexibility to quantify low levels of sIgE.

On the other hand, we compared the ability of our assay to identify allergic and non-allergic individuals. Analysis of the controls (non-allergic patients) shows that hmCLIA, using the standards identifies all the control samples as negative, as the results obtained are below the LOD. The absence of false positives reveals the excellent specificity (100\%) of the hmCLIA method.

Regarding the sensitivity of the in vitro test, the hmCLIA method identified as positive 23 out of the 35 positive samples $(66 \%)$, while ImmunoCAP detected $10(28 \%)$. In order to compare hmCLIA with the reference method at the diagnostic level, the correlation between them was made using the samples detected as positive by both assays. It is worth mentioning that all the positive results given by the ImmunoCAP were also positive by hmCLIA. Receiver Operating Characteristic (ROC) (Figure 4D) analysis showed good area under the curve for hmCLIA. Indeed, both in vitro tests showed high diagnostic specificity since no false positives were detected. In summary, the clinical sensitivity of the developed assay was significantly better, doubling that of the ImmunoCAP assay, what allowed the identification of more positive allergic patients.

\section{Discussion}

Here we showed that the use of selective bispecific binanobodies as synthetic standards is a relevant alternative to quantify specific IgE, improving the performances of in vitro tests for penicillin G allergy testing. This might contribute to the harmonization of this diagnosis and facilitate the appropriate delabeling of patients categorized as allergic to penicillin, thereby reducing the spread of antibiotic resistance and healthcare costs and optimizing patient outcomes. These standards are robust and highly standardized calibrators 
that are easy and inexpensive to produce. Indeed, rBBNs can be reproduced indefinitely in any laboratory with identical characteristics requiring only their sequence. This circumvents the issues of availability and reproducibility related to the use of pooled human sera and defibrinated plasma, in addition to avoiding potential risks of transmission of infectious diseases. This positions rBBNs as advantageous standard material for inter-laboratory comparison studies, allowing verification of whether the methods produce results that agree with the reference one, providing the basis for certificates of quantitative analysis on a given material. In summary, we believe that the work presented here represents a major step towards the standardization of in vitro tests for allergen-specific IgE antibody. This might support the reliable quantification of the level of $\operatorname{sIgE}$ directly to establish a more precise RAST classification. In addition, the strategy of producing this generation of calibrators with different specificities would allow more precise diagnostics to successfully tackle drug allergy delabeling to other antibiotics.

\section{References}

1. Park KH, Lee J, Sim DW, Lee SC. Comparison of singleplex specific ige detection immunoassays: ImmunoCAP Phadia 250 and Immulite 2000 3gAllergy. Ann Lab Med . 2018;38(1):23-31.

2. Casas ML, Esteban Á, González-Muñoz M, Labrador-Horrillo M, Pascal M, Teniente-Serra A. VALIDA project: Validation of allergy in vitro diagnostics assays (Tools and recommendations for the assessment of in vitro tests in the diagnosis of allergy). Adv Lab Med / Av en Med Lab . Published online 2020:1-10.

3. Lucassen R, Fooke M, Kleine-Tebbe J, Mahler M. Development and evaluation of a rapid assay for the diagnosis of immunoglobulin E-mediated type I allergies. J Investig Allergol Clin Immunol . 2008;18(3):223224.

4. Goikoetxea MJ, Sanz ML, García BE, Mayorga C, Longo N, Gamboa PM. Recommendations for the use of in vitro methods to detect specific immunoglobulin E: Are they comparable? J Investig Allergol Clin Immunol . 2013;23(7):448-454.

5. Kleine-Tebbe J, Poulsen LK, Hamilton RG. Quality management in IgE-based allergy diagnostics. LaboratoriumsMedizin . 2016;40(2):81-96.

6. Thorpe SJ, Heath A, Fox B, Patel D, Egner W. The 3rd International Standard for serum IgE: International collaborative study to evaluate a candidate preparation. Clin Chem Lab Med . 2014;52(9):1283-1289.

7. Juárez MJ, Ibañez-Echevarria E, Hernández-Fernández de Rojas D, Maquieira Á, Morais S. Multiplexed analytical approaches to beta-lactam allergy in vitro testing standardization. Anal Chim Acta . $2021 ; 1173: 338656$.

8. Quintero-Campos P, Juárez MJ, Morais S, Maquieira Á. Multiparametric Highly Sensitive Chemiluminescence Immunoassay for Quantification of $\beta$-Lactam-Specific Immunoglobulin E. Anal Chem . 2020;92(21):14608-14615.

9. Smurthwaite L, Durham SR. Local ige synthesis in allergic rhinitis and asthma. Curr Allergy Asthma Rep . 2002;2(3):231-238.

10. Harding FA, Stickler MM, Razo J, DuBridge RB. The immunogenicity of humanized and fully human antibodies: Residual immunogenicity resides in the CDR regions. MAbs . 2010;2(3):256-265.

11. Tu B, Ziemann RN, Tieman BC, et al. Generation and characterization of chimeric antibodies against NS3, NS4, NS5, and core antigens of hepatitis C virus. Clin Vaccine Immunol . 2010;17(6):1040-1047.

12. Mabry R, Lewis KE, Moore M, et al. Engineering of stable bispecific antibodies targeting IL-17A and IL-23. Protein Eng Des Sel . 2010;23(3):115-127.

13. Gonzalez-Sapienza G, Rossotti MA, Tabares-da Rosa S. Single-Domain Antibodies As Versatile Affinity Reagents for Analytical and Diagnostic Applications. Front Immunol . 2017;8.

14. Muyldermans S. Nanobodies: Natural single-domain antibodies. Annu Rev Biochem . 2013;82:775-797. 
15. Kim HJ, McCoy MR, Majkova Z, et al. Isolation of alpaca anti-hapten heavy chain single domain antibodies for development of sensitive immunoassay. Anal Chem . 2012;84(2):1165-1171.

16. Lorimer IAJ, Keppler-Hafkemeyer A, Beers RA, Pegram CN, Bigner DD, Pastan I. Recombinant immunotoxins specific for a mutant epidermal growth factor receptor: Targeting with a single chain antibody variable domain isolated by phage display. Proc Natl Acad Sci U S A . 1996;93(25):14815-14820.

17. Kruger NJ. The Bradford Method. Basic Protein Pept Protoc . Published online 2009:17-24.

Table 1: Specific IgE to Penicillin G determined by hmCLIA and ImmunoCAP (ICAP).

\begin{tabular}{|c|c|c|c|c|c|}
\hline & Calibration & Calibration & Calibration & Calibration & \\
\hline \multirow{3}{*}{ Patient } & WHO & WHO & Binanobody & Binanobody & \\
\hline & ICAP & CLIA & hmCLIA & hmCLIA* & Recovery (\%) \\
\hline & $(\mathrm{IU} / \mathrm{mL})$ & $(\mathrm{IU} / \mathbf{m L})$ & $(\mathrm{PSU} / \mathrm{mL})$ & $(\mathrm{PSU} / \mathrm{mL})$ & \\
\hline 1 & $<0.1$ & $0.6 \pm 0.1$ & $7.1 \pm 0.4$ & $2.4 \pm 0.1$ & - \\
\hline 2 & 3.5 & $0.7 \pm 0.1$ & $9.5 \pm 0.8$ & $3.2 \pm 0.3$ & 91 \\
\hline 3 & $<0.1$ & $<0.1$ & $<0.02$ & $<0.02$ & - \\
\hline 4 & 13.6 & $2.5 \pm 2.5$ & $43.1 \pm 0.7$ & $14.4 \pm 0.2$ & 106 \\
\hline 5 & $<0.1$ & $<0.1$ & $<0.02$ & $<0.02$ & - \\
\hline 6 & $<0.1$ & $<0.1$ & $<0.02$ & $<0.02$ & - \\
\hline 7 & $<0.1$ & $0.9 \pm 0.1$ & $12.6 \pm 0.8$ & $4.2 \pm 0.3$ & - \\
\hline 8 & $<0.1$ & $0.3 \pm 0.1$ & $3.7 \pm 0.1$ & $1.2 \pm 0.1$ & - \\
\hline 9 & 2.2 & $0.9 \pm 0.1$ & $9.7 \pm 0.6$ & $3.2 \pm 0.2$ & 148 \\
\hline 10 & $<0.1$ & $<0.1$ & $<0.02$ & $<0.02$ & - \\
\hline 11 & $<0.1$ & $<0.1$ & $<0.02$ & $<0.02$ & - \\
\hline 12 & $<0.1$ & $2.7 \pm 0.1$ & $47.3 \pm 0.4$ & $15.8 \pm 0.2$ & - \\
\hline 13 & 10.0 & $1.9 \pm 0.2$ & $32.4 \pm 3.0$ & $10.8 \pm 1.0$ & 108 \\
\hline 14 & $<0.1$ & $0.8 \pm 0.1$ & $11.7 \pm 0.2$ & $3.9 \pm 0.1$ & - \\
\hline 15 & $<0.1$ & $<0.1$ & $<0.02$ & $<0.02$ & - \\
\hline 16 & 1.8 & $0.5 \pm 0.1$ & $6.5 \pm 0.4$ & $2.2 \pm 0.1$ & 118 \\
\hline 17 & $<0.1$ & $0.6 \pm 0.2$ & $8.1 \pm 0.2$ & $2.7 \pm 0.1$ & - \\
\hline 18 & $<0.1$ & $0.6 \pm 0.1$ & $7.4 \pm 0.1$ & $2.5 \pm 0.1$ & - \\
\hline 19 & $<0.1$ & $0.3 \pm 0.1$ & $3.2 \pm 0.2$ & $1.1 \pm 0.1$ & - \\
\hline 20 & 1.8 & $0.5 \pm 0.1$ & $6.0 \pm 0.2$ & $2.0 \pm 0.1$ & 109 \\
\hline 21 & 2.2 & $0.5 \pm 0.1$ & $6.7 \pm 0.4$ & $2.2 \pm 0.1$ & 112 \\
\hline 22 & $<0.1$ & $<0.1$ & $<0.02$ & $<0.02$ & - \\
\hline 23 & $<0.1$ & $0.9 \pm 0.1$ & $13.2 \pm 0.1$ & $4.4 \pm 0.1$ & - \\
\hline 24 & $<0.1$ & $0.3 \pm 0.1$ & $3.6 \pm 0.3$ & $1.2 \pm 0.1$ & - \\
\hline 25 & $<0.1$ & $<0.1$ & $<0.02$ & $<0.02$ & - \\
\hline 26 & $<0.1$ & $<0.1$ & $<0.02$ & $<0.02$ & - \\
\hline 27 & 0.4 & $0.2 \pm 0.1$ & $1.2 \pm 0.1$ & $0.4 \pm 0.1$ & 92 \\
\hline 28 & 3.0 & $1.0 \pm 0.1$ & $12.2 \pm 0.4$ & $4.1 \pm 0.1$ & 136 \\
\hline 29 & $<0.1$ & $<0.1$ & $<0.02$ & $<0.02$ & - \\
\hline 30 & $<0.1$ & $1.3 \pm 0.1$ & $21.0 \pm 0.2$ & $7.0 \pm 0.1$ & - \\
\hline 31 & $<0.1$ & $<0.1$ & $<0.02$ & $<0.02$ & - \\
\hline 32 & $<0.1$ & $<0.1$ & $<0.02$ & $<0.02$ & - \\
\hline 33 & 91.1 & $40.9 \pm 0.9$ & $270.5 \pm 6.0$ & $90.2 \pm 2.0$ & 99 \\
\hline 34 & $<0.1$ & $0.6 \pm 0.1$ & $8.0 \pm 0.5$ & $2.7 \pm 0.2$ & - \\
\hline 35 & $<0.1$ & $0.5 \pm 0.1$ & $5.3 \pm 0.1$ & $1.8 \pm 0.1$ & - \\
\hline $36^{\mathrm{a}}$ & 12.5 & $4.5 \pm 0.4$ & $41.1 \pm 3.8$ & $13.3 \pm 1.1$ & 106 \\
\hline
\end{tabular}


*The concentrations are multiplied by a factor of $1 / 3$ to get those obtained by ImmunoCAP. ${ }^{\text {a Pooled-sample }}$ from patients $2,4,9,13,16,20,21,27,28$ and 33 , using equal volume of each.
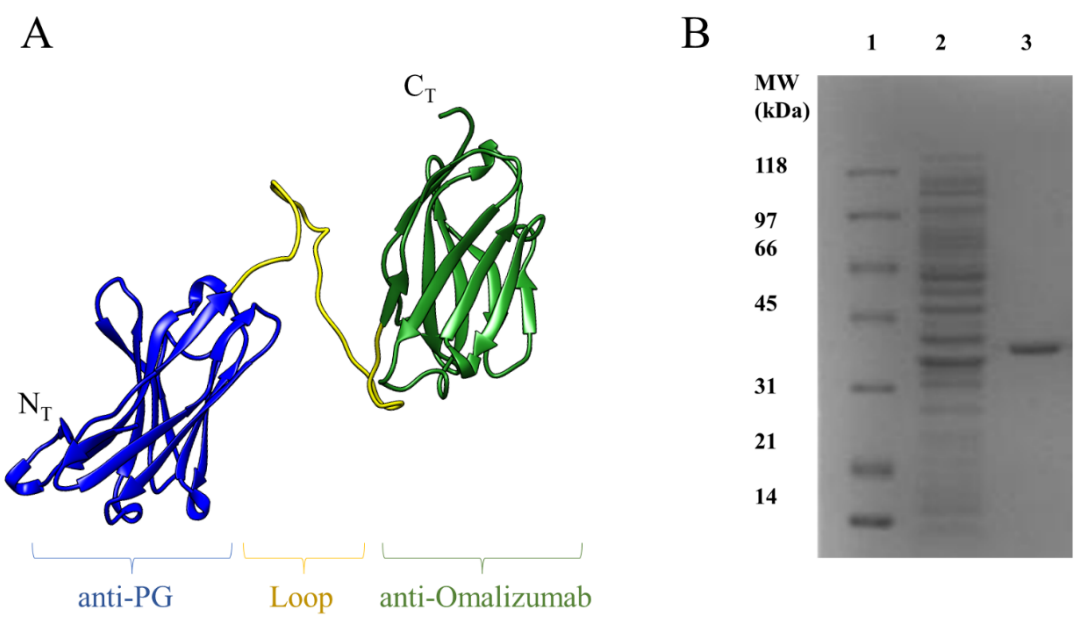

Figure 1. [A] Model structure of the rBBN. On the left (blue), the nanobody that targets the antigenic determinant, on the right (green) the one that binds the paratope of Omalizumab. [B]Purification results 12 \% SDS-PAGE: (1) Ladder (2) Purification Flow Through (3) Bispecific binanobody with a molecular weight of $35 \mathrm{kDa}$.

\section{Hosted file}

image2.emf available at https://authorea.com/users/449386/articles/547921-tacklingpenicillin-allergy-delabeling-with-ultra-sensitive-in-vitro-test-and-human-likespecific-ige-standards

Figure 2. [A] Curve of functionality of the bispecific binanobody. [B] Results of thermofluor assay for rBBN alone and bound to conjugate H1-PG. Representation of the melting curve using the first derivative $-(\mathrm{dRFU}) / \mathrm{dT}$ of the raw data. The Tm is the apex. [C] Blue Native Page: (1) rBBN $1 \mu \mathrm{M}$. (2) rBBN $3 \mu \mathrm{M}$. (3) H1-PG $1 \mu \mathrm{M}$. (4) H1-PG $1 \mu \mathrm{M}+\operatorname{rBBN} 1 \mu \mathrm{M}$. (5) H1-PG $1 \mu \mathrm{M}+\operatorname{rBBN} 3 \mu \mathrm{M}$. (6) Omalizumab $1 \mu \mathrm{M}$. (7) $\mathrm{rBBN} 1 \mu \mathrm{M}+$ Omalizumab $1 \mu \mathrm{M}$. (8) H1-PG $1 \mu \mathrm{M}+\operatorname{rBBN} 1 \mu \mathrm{M}+$ Omalizumab $1 \mu \mathrm{M}$. (9) H1-PG 1 $\mu \mathrm{M}+\mathrm{rBBN} 3 \mu \mathrm{M}+$ Omalizumab $1 \mu \mathrm{M}$.

\section{Hosted file}

image7.emf available at https://authorea.com/users/449386/articles/547921-tacklingpenicillin-allergy-delabeling-with-ultra-sensitive-in-vitro-test-and-human-likespecific-ige-standards

Figure 3. [A] Scheme of the homologous chemiluminescence immunoassay for the determination of specific IgE to penicillin $G$ in human serum, using the $\mathrm{rBBN}$ as calibrator. [B] Binanobody calibration curve.

\section{Hosted file}

image8.emf available at https://authorea.com/users/449386/articles/547921-tacklingpenicillin-allergy-delabeling-with-ultra-sensitive-in-vitro-test-and-human-likespecific-ige-standards 
Figure 4: [A] Scatter diagram and regression line of inter-method comparison between homologous calibration $(\mathrm{rBBN})$ and heterologous calibration (WHO). [B] Scatter diagram and regression line of inter-method comparison between hmCLIA and ImmunoCAP.[C] Dilution linearity study, using the pooled serum sample and a pool of control serum samples as diluent. Dotted lines represent the $99 \%$ confidence interval. [D] ROC analysis representing the area under the curve (AUC). Sensitivity and specificity of the hmCLIA as compared against ImmunoCAP for penicillin $\mathrm{G}(\mathrm{n}=65$ values). 